\section{Gastroösophageales Karzinom: Klinische Remission vor Operation ohne Aussagekraft}

Patienten mit lokalisiertem gastroösophagealem Karzinom (GEC) stellen eine Operation nach erfolgreicher neoadjuvanter Chemoradiotherapie häufig infrage. Die Therapiergebnisse einer Chemoradiatio ohne anschließende Operation sind jedoch heterogen. Ist eine komplette Remission ein möglicher Prädiktor für die weitere Therapie?

I einer retrospektiven Analyse untersuchten Kollegen vom MD Anderson Cancer Center in Houston TX/USA die Assoziation zwischen einer präoperativen klinischer kompletter Remission (clinCR) und einer pathologischen kompletten Remission (pathCR) nach der Operation bei Patienten mit lokalisiertem GEC. Eine clinCR lag vor, wenn nach der Chemoradiatio, aber noch vor der Operation, die endoskopische Biopsie negativ ist und sich in der Positronenemissionstomografie (PET) Fluorodeoxyglukose (FDG) nur in physiologischer Menge im Zielgewebe anreicherte. Eine pathCR war definiert durch Abwe- senheit von Krebszellen im operativ gewonnenen Resektat. In die Untersuchung gingen die Daten von 284 Patienten ein, die zwischen 2002 und 2010 wegen eines gastroösophagealem Karzinom eine Chemoradiatio mit anschlieBender Ösophagektomie behandelt worden waren.

Von den 284 Patienten erreichten 218 (77\%) eine clinCR. Von diesen 218 Patienten war das Resektat aber nur bei 67 ohne Tumorzellen (pathCR: $31 \%$ ). Von den 66 Patienten, die keine clinCR erreichten, hatten lediglich zwei (3\%) eine pathCR. Dieses Ergebnisse implizieren, dass 151 Patienten $(69,3 \%)$ der 218 Pati- enten mit clin CR keine pathCR erreichten. Die Rate der pathCP war damit bei Patienten mit clinCR signifikant verschieden von denen, die keine clinCR erreichten $(\mathrm{p}<0,001)$. Die Sensitivität der clinCR für die pathCR betrug zwar $97,1 \%$, die Spezifität war aber mit $29,8 \%$ ausgesprochen niedrig.

Fazit: Bei GEC-Patienten korreliert die klinische Komplettremission nach Chemoradiotherapie nicht besonders stark mit der pathologischen Komplettremission im Resektat. Die Spezifität der clinCR für eine pathCR ist zu gering, um eine Empfehlung für das weitere therapeutische Vorgehen abgeben zu können. Auch Patienten mit einer clinCR sollten sich deshalb nach wie vor einer geplanten Operation unterziehen.

Barbara Kreutzkamp

Cheedella NKS et al. Association between clinical complete response and pathological complete response after preoperative chemoradiation in patients with gastroesophageal cancer: analysis in a large cohort. Ann Oncol. 2012 Dec 17. [Epub ahead of print]

\section{Second-Line-Sunitinib bei GIST: Hypertonie als Prädiktor für den Behandlungserfolg}

\begin{abstract}
Aktivierende Mutationen im KIT- oder PDGFRA-Gen sind ein starker Prädiktor für das Ansprechen einer Sunitinib-Therapie in der First-Line. Bisher fehlen allerdings Biomarker die in weiteren Therapielinien ein Vorhersage erlauben. Ein möglicher Kandidat: die medikamenteninduzierte Hypertonie.
\end{abstract}

\footnotetext{
$\mathrm{n}$ der Behandlung von metastasierten Gastrointestinalen Stromatumoren (GIST) führt der Tyrosinkinaseinhibitor Sunitinib in der First-Line bei über $80 \%$ und in der Second-Line bei über $60 \%$ der Patienten zur Krankheitskontrolle. In der First-Line-Therapie kann die Response anhand der Tumor-Genotypisierung gut vorhergesagt werden. Für die Second-Line bietet sich möglicherweise die medikamenteninduzierte Hypertonie als Biomarker für die Therapieresponse an. Eine ganze Reihe von gezielten Therapeutika, so auch Sunitinib, blockieren den VEGF (vascular endothelial growth factor)-Rezeptor 2, erhöhen dadurch den peripheren vasku-
}

lären Widerstand und induzieren eine Hypertonie.

In einer retrospektiven Analyse mit gepoolten Daten dreier Studien zur Sunitinib-Therapie bei fortgeschrittenem GIST wurden Korrelationen zwischen einer Sunitinib-assoziierten Hypertonie und der Antitumoraktivität (n $=319)$ sowie die Sicherheit $(n=1.565)$ untersucht. In multivariaten Analysen verglich man Patienten mit Bluthochdruck ( $\geq 140 \mathrm{mmHg}$ systolisch und/oder $\geq 90 \mathrm{mmHg}$ diastolisch) und ohne Hochdruck während der Sunitinib-Behandlung, die Time-to-event-Endpunkte wurden mit Kaplan-Meier-Modellen geschätzt.
Eine Sunitinib-assoziierte Hypertonie korrelierte mit Verbesserungen bei objektiven Responseraten, der Zeit bis zum Progress, dem progressionsfreien Überleben und dem Gesamtüberleben. Diese Zusammenhänge blieben auch in multivariaten und Landmark-Analysen signifikant. Die Inzidenz unerwünschter Wirkungen durch die medikamenteninduzierte Hypertonie war gering, kardiovaskuläre Nebenwirkungen traten bei den Hypertonie-Patienten etwas häufiger auf.

Fazit: Bei Patienten mit fortgeschrittenem GIST korreliert die Sunitinib-assoziierte Hypertonie mit einem besseren Therapieergebnis.

Unerwünschte kardiovaskuläre, zerebrovaskuläre und okuläre Hypertonieinduzierte Ereignisse waren vergleichsweise gering und ließen sich gut kontrollieren.

Barbara Kreutzkamp

George S et al. Hypertension as a potential biomarker of efficacy in patients with gastrointestinal stromal tumor treated with sunitinib. Ann Oncol. 2012;23(12):3180-7. 